\title{
Les dynamiques organisationnelles dans la maintenance des matériels roulants
}

Organizational dynamics in the maintenance of the rolling stock

Gilbert de Terssac et Karine Lalande

\section{OpenEdition}

\section{Journals}

Édition électronique

URL : https://journals.openedition.org/rhcf/1767

DOI : 10.4000/rhcf.1767

\section{Éditeur}

Rails \& histoire

\section{Édition imprimée}

Date de publication : 1 décembre 2003

Pagination : 222-235

ISBN : 0996-9403

ISSN : 0996-9403

\section{Référence électronique}

Gilbert de Terssac et Karine Lalande, "Les dynamiques organisationnelles dans la maintenance des matériels roulants », Revue d'histoire des chemins de fer [En ligne], 28-29 | 2003, mis en ligne le 19 décembre 2014, consulté le 22 avril 2022. URL : http://journals.openedition.org/rhcf/1767 ; DOI : https://doi.org/10.4000/rhcf.1767 


\section{Les dynamiques organisationnelles dans la maintenance des matériels roulants ${ }^{1}$}

La recherche que nous présentons porte sur la modernisation de l'organisation de l'entretien du matériel roulant à la SNCF, de 1953 à $1992^{2}$. L'histoire de cette modernisation commence au début des années 1950, quand la SNCF décide d'adopter un nouveau type de courant pour commencer à électrifier le nord-est de son réseau : le courant monophasé. À l'époque, d'autres parties du réseau, le Sud-Ouest et le Sud-Est, sont déjà électrifiées, mais en courant continu. L'arrivée, en 1954, des machines à traction électrique en courant monophasé constitue le point de départ d'une réorganisation de l'ensemble des activités professionnelles de ce secteur. L'entretien des premières machines électriques en courant monophasé sera l'occasion et le point de départ d'un changement organisationnel, sans précédent, des pratiques d'entretien, en même temps qu'il constitue une innovation technologique majeure pour l'entreprise qui va entrer alors dans une période d'unification et de

1- Nous reprenons ici, pour l'essentiel, la formalisation que nous avons proposée dans notre article : Gilbert de Terssac "Travail d'Organisation et travail de régulation », in Gilbert de Terssac (sous la dir. de), La Théorie de la régulation sociale de J.-D. Reynaud: débats et prolongements, Paris, La Découverte, 2003.

2- Cette recherche a été réalisée, de 1996 à 1998, à la demande de la direction du Matériel et de la Traction et de la direction des Ressources humaines de la SNCF. L'appel à projet était intitulé : «Recherche sociologique et historique sur l'évolution de l'organisation de la maintenance du matériel roulant. »Cette recherche s'est déroulée au sein du Centre d'études et de recherches sur travail, organisation et pouvoir (CERTOP, UMR 5044 du CNRS) de l'université Toulouse-Le Mirail. La recherche a donné lieu, tout d'abord, à un rapport de recherche, intitulé «La dynamique de construction de l'organisation de la maintenance du matériel roulant : 1954-1992» (par G. de Terssac et K. Lalande, 1998). Elle a donné lieu ensuite à une thèse de doctorat en sociologie intitulée «Le travail d'organisation dans la maintenance » (par K. Lalande, 1999). Enfin, un ouvrage est venu clôturer ce programme : Gilbert de Terssac ; Karine Lalande, Du train à vapeur au TGV. Sociologie du travail d'organisation, Paris, PUF, 2002.

Nos remerciements vont tout d'abord à l'ensemble des personnes qui ont accepté de nous recevoir et de nous livrer une partie de leur expérience. Ils vont aussi aux membres du groupe de pilotage de cette recherche et en particulier à J.-Michel Mailfer et à Jacques Desmis qui ont suivi cette recherche en nous en facilitant la réalisation. Enfin, nous tenons à dire tout ce que nous devons à J.-D. Reynaud (professeur émérite au CNAM) dont les remarques et conseils ont inspiré ce texte. 
modernisation ${ }^{3}$. Précisons que l'entretien occupe une place stratégique importante dans l'entreprise, dans la mesure où il faut obtenir dans ce secteur des résultats immédiatement perceptibles : lorsque l'entretien est réussi, ce secteur ne fait pas parler de lui, mais la survenue d'incidents ou d'accidents remet parfois l'entretien sur le devant de la scène.

Nous avons mis en évidence trois phases de cette dynamique organisationnelle ${ }^{4}$. Dans la première phase, de 1953 à 1965, les acteurs doivent inventer un nouveau système d'organisation pour résoudre les problèmes d'entretien liés à une innovation technique et surtout se détacher du système d'organisation en vigueur, celui du temps « de la vapeur ». Dans la deuxième phase, de 1965 à 1975, voire 1980 pour certains établissements, les acteurs généralisent le nouveau schéma d'organisation, formulent les principes généraux, forment un acteur collectif pour servir le projet et prennent le pouvoir pour assurer la «normalisation des établissements ». Au cours de la troisième phase, de 1974 à 1994, le système d'organisation se dégrade et fait l'objet d'une contestation; les acteurs doivent résoudre de nouveaux problèmes: de nouvelles générations de machines équipées d'électronique arrivent (le TGV), les « démarches qualité » doivent être

3- On parle d'une « seconde révolution ferroviaire» (Ribeill, 1992).

4- L'investigation a combiné plusieurs méthodes. Premièrement, des entretiens de deux heures environ avec 121 personnes, enregistrés, transcrits et validés par leurs auteurs. L'échantillon comprend des personnes qui ont travaillé au Matériel, au cours de leur carrière professionnelle, même s'ils occupent d'autres positions au moment de l'entretien. Le recueil de leur témoignage se fait toujours de la même manière : un résumé de la trajectoire professionnelle, la description de l'organisation au moment où ils entrent au Matériel (plus d'un tiers étaient ouvriers à ce moment-là), la manière dont ils ont perçu son évolution, leur contribution et leur position à l'égard de l'organisation. Les 121 personnes sont, au moment de l'étude, retraitées pour certaines, dirigeants en activité pour d'autres ou responsables en établissement, syndicalistes ou dans d'autres postes en dehors du Matériel. La règle du jeu a été le volontariat pour rencontrer l'équipe de recherche et l'anonymat dans la présentation des résultats. Deuxièmement, nous avons recueilli de nombreuses traces écrites : des documents d'organisation généraux, des actes des congrès du Matériel, des articles de dirigeants, des procédures de travail, des données statistiques, des tracts syndicaux. Au total, plus de 1600 pages d'écrits ont été recueillies, analysées et transmises au service responsable de l'archivage des documents sous forme de 10 volumes. Ces écrits constituent des traces pertinentes, non seulement pour les confronter aux témoignages des acteurs, mais surtout pour analyser la manière dont ils codifient la réalité, tentent de donner une image commune de la cible à atteindre, approfondissent l'argumentation, énoncent des principes ou des règles à respecter. Troisièmement, huit établissements ont fait l'objet d'une analyse monographique ayant pour objet de retracer en situation cette dynamique organisationnelle, variable selon les secteurs (dépôt ou atelier). 
mises en œuvre et l'efficacité renforcée. En partant de la caractérisation des différentes phases (1), nous allons retracer cette dynamique qui consiste à produire de nouvelles règles d'entretien (2), à faire émerger de nouveaux acteurs qui s'allient ou s'opposent, entrent en conflit ou négocient (3), en fonction d'une « manière de concevoir » l'entretien et de faire valoir cette conception (4) de l'objet.

\section{Les trois phases de la dynamique organisationnelle}

Nous avons caractérisé cette dynamique en trois phases : « une phase d'expérimentation », une phase de "généralisation », une phase de «différenciation ». La phase « d'expérimentation » est la phase qui rompt avec le système d'entretien des machines à vapeur : les acteurs cherchent à domestiquer une innovation technique par l'invention d'un autre schéma d'organisation. Dans la phase de « généralisation », c'est la normalisation : les porteurs du projet de modernisation vont imposer partout un schéma d'organisation, quel que soit le matériel concerné, des locomotives mais aussi des voitures ou des wagons. La phase de « différenciation » est la phase des projets, des reprises d'initiative. C'est la survie des établissements qui est en jeu. Pour se faire une idée de la tendance de l'époque, il faut rappeler que le nombre d'établissements est passé de 178 en 1973 à 102 en 1993.

\section{La phase "expérimentale " : 1953 à 1965}

La première phase est «expérimentale»: un groupe d'acteurs, que nous appellerons «les inventeurs », se constitue dans un dépôt de l'Est de la France en 1953 pour inventer et mettre au point des manières de travailler différentes de celles de la vapeur, afin de résoudre les problèmes posés par l'entretien des nouvelles machines électriques en courant monophasé. Par l'observation du comportement des machines, les compagnons et leur encadrement immédiat élaborent les lois d'usure des matériels, combinent ces connaissances de terrain avec les savoirs de conception et, ensuite, déposent ces savoirs et ces connaissances dans des règles d'intervention : les savoirs ainsi constitués vont servir de valeur d'arbitrage pour le choix des règles d'intervention appropriées. Ce groupe, minoritaire, est composé de ceux qui tentent de maitriser le fonctionnement des nouvelles machines électriques : il s'agit de quelques dizaines de compagnons et de la hiérarchie engagées dans la maitrise de cette innovation ; pour l'un des promoteurs de cette démarche, il faut délocaliser la production des normes de travail vers le bas, car, dit-il, « l'organisation, c'est la compétence, la compétence, c'est à la base qu'on la trouve ». Ils forment une coalition efficace fondée sur 
la cooptation des membres, leur formation et une structuration en équipes chargées de construire et de faire évoluer les procédures, en fonction du contrôle de leur efficacité. Leur action reste cependant limitée car elle dépend du cadre local (les machines électriques et les dépôts de l'est de la France) et de son caractère, dominé par le mode de travail issu de la vapeur. À la fin de cette première phase (1965), ce groupe comprend quelques centaines de personnes, réparties sur une dizaine de sites.

\section{La phase "de généralisation » : 1965 à 1975-1980}

La seconde phase est celle de la "généralisation » : au cours de cette phase, le référentiel local, issu de la phase précédente, est la source et le moyen de construire le référentiel global ; le groupe porteur de ces règles locales et dominé, que nous appellerons "les modernisateurs", va devenir dominant et être doté du pouvoir d'imposer le schéma local à tous les établissements. On peut résumer cette phase de généralisation par les propos de l'un des dirigeants de l'époque, qui retracent bien l'enjeu de cette «normalisation » : « Ma ligne de conduite, dira un dirigeant, a été de dire premièrement il nous faut une doctrine. Deuxièmement, il faut la formaliser. Troisième point, il faut l'enseigner et quatrième point il faut imposer, si possible de bon gré, sa mise en œuvre sur le terrain. » Le groupe des «modernisateurs » est composé de tous ceux qui se sont " rangés » à la culture normative élaborée au cours de la phase précédente et qui se sont engagés à la propager dans l'ensemble des établissements, en échange d'une promotion. Sa constitution se fait par une socialisation professionnelle consistant à faire passer les personnes par des postes "formateurs $\|^{5}$, avant de faire partie de l'encadrement. De plus, son action locale visant la « normalisation » des établissements selon le schéma d'organisation préconisé est encadrée par le « haut»: les initiatives organisationnelles locales sont désormais régulées par un pouvoir central. Cette centralisation de la régulation se caractérise tout d'abord par la création d'une direction du Matériel qui devient un centre de décision unique capable d'écarter les « récalcitrants » et d'engager une lutte de pouvoirs contre les chefs de service «qui étaient des potentats » ou contre les chefs d'établissement car « il y avait plus d'enthousiasme, dira l'un des promoteurs, chez les compagnons qu'au niveau des dirigeants d'établissement ».

5- Par poste formateur, il faut entendre, (a) les «rapporteurs » chargés d'élaborer les documents, (b) les «instructeurs » chargés de faire partager le schéma d'organisation, (c) les « binômes » pour mettre en œuvre le schéma d'organisation. 


\section{La phase de "différenciation »: 1980-1994}

La troisième phase est celle de la « différenciation » : elle est marquée avant tout par l'apparition du groupe des contestataires que nous appellerons les « innovateurs-projet», qui se constitue contre les «applicateurs zélés » qui appliquent à la lettre le schéma d'organisation sans en garder l'esprit et les valeurs qui le fondaient, comme la production des règles par les compagnons eux-mêmes. Ce groupe « d'innovateursprojet » reprend l'initiative dont il a été privé lors de la phase précédente et agit en quelque sorte contre les « règles bureaucratiques » que les « applicateurs zélés » veulent conserver. Ils élaborent des projets locaux : certains visent la mise en œuvre, au sein d'un établissement, d'une démarche qualité incitée par la direction centrale; d'autres projets sont dédiés à l'établissement, comme l'enrichissement des tâches, le raccourcissement de la ligne hiérarchique, la mise en place « de groupes autonomes » ou d'un système de "maintenance personnalisée selon l'état» (MAPSE) du matériel. Ce groupe comprend des acteurs des établissements, mais aussi des dirigeants nationaux ${ }^{6}$ soucieux de voir les établissements reprendre de l'initiative, là où ils avaient le sentiment que la situation était bloquée ; soucieux aussi de compter sur de nouveaux alliés pour mettre en place des innovations techniques ou touchant les règles de travail.

\section{Les " règles émergentes " contre les " règles en place "}

Le TO - travail d'organisation, en opposition avec l'Organisation scientifique du travail, OST - est avant tout un travail de production de règles et un effort pour rendre ces règles communes : comment un système de règles dominé peut-il devenir dominant? Dans notre recherche, les règles émergentes, d'abord inventées, puis étendues à tous les établissements et enfin retravaillées pour les leur adapter, ne résultent pas d'un compromis les «règles en place » du «système vapeur»: il s'agit d'un système de règles "émergentes » élaborées pour servir le projet de modernisation et qui prennent une figure différente à chaque stade de développement du projet.

\section{Des règles " efficaces " contre les règles détériorées}

Dans la phase expérimentale, les « règles d'efficacité » sont élaborées pour entretenir les machines électriques et elles sont conçues

6- La direction centrale et une partie de la ligne hiérarchique des établissements se rendent compte du «blocage » de l'initiative dès 1980, lors du congrès de Blois, et ce congrès marque le retour vers l'initiative et conduit petit à petit à l'extinction du corps des « applicateurs zélés ». 
pour remplacer les règles du système « vapeur ». Les transformations des règles pratiques portent sur les temps alloués qui sont remplacés par de l'autocontrôle, sur les petits carnets du compagnon qui disparaissent au profit de procédures écrites, sur l'apprentissage sur le terrain et par contact qui se déroule désormais en salle, sur le pouvoir de décision centralisé par les experts qui est délocalisé vers les compagnons en charge d'écrire les procédures (on est dans les années cinquante). Autrement dit, les règles de travail en place ne sont ni reprises, ni réaménagées car elles sont considérées par les inventeurs comme des « règles dégradées » peu efficaces. En même temps que les acteurs inventent de nouvelles règles pratiques, ils inventent des règles du jeu social différentes, fondées sur une remise en cause de la division du travail et de la confiscation des savoirs par les experts qui s'étaient arrogés, au temps de la vapeur, le pouvoir de définir et de contrôler les normes de travail : élaborer des règles d'efficacité, c'est se mettre d'accord sur le problème à résoudre en délégitimant les procédures en place, s'engager pour le résoudre en inventant des règles, s'entendre pour considérer que le compagnon doit élaborer les procédures, car il est détenteur de savoirs et de savoir-faire au détriment des experts rendus inutiles.

\section{Des règles " de contrôle " contre les règles " convention- nelles»}

Dans la phase de " généralisation », les modernistes étendent les règles d'efficacité à tous les matériels et à tous les établissements ; du coup, ils transforment ces règles autonomes en « règle de contrôle » en vue d'homogénéiser la spécialisation, la formation, l'élaboration des documents et la structuration des équipes. Pour imposer les règles pratiques issues de la phase précédente à tous les acteurs, les modernisateurs élaborent de nouvelles règles du jeu social. Car organiser les établissements à distance et selon une voie unique et impérieuse, ce n'est pas seulement harmoniser des pratiques ; c'est aussi condamner les manières de faire en vigueur, s'attaquer aux fondements du système social et remplacer les règles en vigueur au cours de cette phase qui sont les « règles conventionnelles » liées au temps de la vapeur et qui perdurent dans la plupart des établissements. C'est pourquoi les modernistes élaborent des " règles de contrôle ", afin d'orienter dans la même direction leurs actions et de coordonner leurs initiatives. Ces règles visent à élaborer la « doctrine » précisant les valeurs à défendre et les orientations à respecter, à encadrer de manière centralisée l'initiative locale par une prise de pouvoir, à créer les institutions permettant d'exercer ce contrôle et de diffuser la doctrine, à récompenser, par une promotion, 
ceux qui s'engagent dans la normalisation et qui occupent les postes " formateurs » permettant de tester leur loyauté, à écarter la hiérarchie locale qui s'oppose à la progression du projet.

\section{Des règles de "coopération" contre les règles " bureaucra- tiques"}

Cette création de règles pour remplacer les règles en place est moins évidente lors de la phase de différenciation. Pourtant, les acteurs doivent faire face à de nouveaux problèmes d'efficacité, liés à des transformations du contexte : de nouvelles générations de machines arrivent comme le TGV ou des machines dotées d'électronique embarquée, les relations avec le client se structurent davantage autour de la qualité, les acteurs locaux issus des collectivités locales ont leur mot à dire sur la politique de transport qu'ils soutiennent financièrement, le personnel est mieux formé et plus exigeant au regard du travail qui lui est demandé. De plus, un climat de «blocage » et un sentiment de perte d'initiative s'étaient installés tant auprès des dirigeants nationaux que des hiérarchies locales : les règles en vigueur, pourtant mises en place par les modernistes, sont considérées par certains comme inefficaces, moyennes, imposées, ne tenant pas compte des spécificités locales, tant des matériels que des situations ou des personnes; un vent de critique s'installe à propos des règles du nouveau schéma d'organisation ${ }^{7}$, considérées comme ayant été appliquées sans discernement, « à la lettre », transformant les modernistes en bureaucrates. Dès lors, au cours de cette phase les innovateurs-projet élaborent et mettent en œuvre des «règles de coopération» : ils élaborent ces règles autour d'un projet spécifique tel que l'allégement d'une structure hiérarchique, un regroupement des tâches autour d'un produit et non par métier, une démarche qualité, voire la modification d'un atelier d'entretien pour accueillir des TGV. Ces règles de coopération précisent les contours d'un projet élaboré collectivement tant par les acteurs d'un établissement précis qu'avec les directions centrales ou régionales dont ils dépendent.

7- Bien que les acteurs interviewés appartiennent à des générations différentes et que leur contribution varie en fonction du moment de leur intégration dans l'entreprise, la quasi-totalité de nos interlocuteurs s'accorde sur ce point. La génération des inventeurs pense que le système qu'ils ont inventé n'a pas été repris dans l'intégralité de ses caractéristiques, notamment du point de vue du temps nécessaire pour que les compagnons participent à l'élaboration des règles de travail ou s'approprient le système nouveau. Les modernistes et, en particulier, les dirigeants ont très tôt pris conscience du fait «que le système leur échappait», qu'il y avait des dérives ; enfin, les innovateurs-projet constituent la génération qui a subi le projet plus qu'elle ne l'a voulu : ils se sont retournés contre les déviations. 
Elles sont validées par l'acceptation du projet et par la coalition qui se forme pour stabiliser les attentes des acteurs en présence : ils s'unissent autour de l'examen d'un problème lié à la survie ou la pérennisation d'un établissement dont le projet local est la solution.

\section{Acteur collectif, opposition et subordination}

La réorganisation du secteur de l'entretien des matériels brouille la vision pourtant claire de deux collectifs distincts et séparés selon une logique d'opposition : elle introduit une crise de l'efficacité et de la légitimité de cette division entre dirigeants et dirigés, entre hiérarchie et subordonnés, entre décideurs et exécutants. Elle ne va pas abolir cette division et ces oppositions mais les déplacer, faire éclater ces deux collectifs et les disperser dans de nouveaux collectifs. Le collectif des « dirigeants » ou de la hiérarchie est déstabilisé et désolidarisé par le projet de modernisation : il n'a plus le monopole de la production normative et ne peut plus être considéré comme l'acteur légitime garant de l'ordre et de la raison, puisqu'à côté de lui et " tout contre » va se mettre en place un autre acteur qui va lui disputer sa prétention à légiférer ; ce sont les inventeurs d'abord, puis les modernistes, enfin les innovateurs-projet. De même, le collectif des «subordonnés » ou des exécutants va se fissurer à l'occasion de ce projet de modernisation : il n'a plus le monopole de l'opposition qui va lui être disputé par certaines hiérarchies locales qui vont s'opposer au projet. De plus, les acteurs qui, par leur position dans le processus de travail, devraient s'opposer à la modernisation vont changer de camp et s'associer au moins partiellement au projet, plus que s'y opposer : c'est le cas des compagnons qui y ont vu l'opportunité d'améliorer leur conditions de travail, leur formation, leurs relations de travail ; mêmes les syndicats seront déstabilisés et ne réussiront pas à s'opposer au projet, faute d'arguments et de combattants.

\section{Les « inventeurs » sous le regard des " sceptiques»}

Dans la phase « expérimentale », le groupe des « inventeurs » est dominé par le groupe des «sceptiques », majoritaire ; ce dernier est composé de ceux qui voient arriver dans leurs établissements ce nouveau schéma, sans pour autant s'y opposer. Ils attendent, hésitent à s'engager, n'anticipent pas ou ne perçoivent pas l'impact qu'aura ce projet, ni le statut qu'il aura dans l'avenir. Ils conservent leur mode de travail « de type vapeur» et ne sont pas dépendants de l'action menée par les autres. Ils veulent voir les bénéfices, espèrent en tirer les avantages, évaluent les procédures et comparent les résultats : il s'agit des agents 
et de la hiérarchie de l'établissement dans lequel s'expérimente le nouveau schéma ; ils ne perçoivent pas le fait que l'univers ordonné de leurs pratiques va être déstabilisé ou ils considèrent que l'expérience s'arrêtera aux machines électriques.

\section{Les « modernisateurs " s'opposent aux « conventionnalistes "}

Dans la phase de "généralisation », les «modernisateurs » vont entrer en conflit avec les « conventionnalistes » qui, en s'abritant sur le dispositif « vapeur » encore dominant, tentent de s'opposer aux « modernisateurs » : il est composé des hiérarchies d'établissement ou des réseaux, mais aussi des compagnons qui s'opposent à la remise en cause du système vapeur. Ils sont soudés autour du schéma d'organisation « vapeur » qui a un effet stabilisant ; leur comportement est encadré par les règles coutumières et traditionnelles en vigueur. Ils sentent bien la menace qui pèse sur leurs prérogatives et s'opposent au nouveau système qui va balayer les avantages qu'ils tiraient du système en place. Cette menace est d'autant mieux perçue que l'un des «modernistes » raconte qu'«il y a une période où, pour lancer l'organisation, il fallait compter sur des hommes et il y a eu des drames : ceux qui en toute bonne foi ne voulaient pas, ont été écartés ».

\section{Les « innovateurs-projet » contre les nouveaux « bureaucrates »}

Enfin, dans la phase de différenciation les «innovateurs-projet» vont s'opposer aux « modernisateurs » qui se sont transformés en vrais «bureaucrates » : les premiers qualifient les seconds d'« applicateurs zélés ${ }^{8}$ » qui tentent de brider toute initiative, en s'abritant derrière des règles bureaucratiques. Ce groupe de bureaucrates est composé essentiellement

8- Les règles élaborées au cours de la généralisation ont été construites par les modernisateurs pour normaliser les établissements. Mais ces règles qui constituaient une contrainte pour les établissements ont été utilisées par les « applicateurs zélés » comme une ressource au service de leur stratégie. Le système d'action des modernistes a été déplacé puisque les « applicateurs zélés » ont tourné ces règles à leur avantage et ont optimisé leur choix, en laissant de côté le sens de la règle défini par les modernistes et l'esprit de l'action qu'ils avaient engagée. Des « applicateurs zélés », les acteurs diront tout d'abord qu'ils n'avaient pas compris la démarche : «il y a des gens qui venaient prêcher des choses qu'ils n'avaient pas forcément bien comprises et qui en rajoutaient dans les exigences vis-à-vis des établissements, croyant faire plaisir au chef. » De plus, ils affichaient une loyauté a minima: "faire comprendre la méthode, ce n'est pas simplement en imposant, il faut expliquer [...]. Les gens n'étaient pas convaincus de ce qu'ils faisaient ». Enfin, il leur est reproché d'avoir utilisé la méthode à des fins personnelles : « ils avaient compris qu'il suffisait d'apprendre un discours pour être reconnu [...]. En acceptant les principes d'organisation du matériel, ils pouvaient faire une carrière professionnelle. » 
d'un encadrement intermédiaire formé par les «modernistes » dont ils se sont détachés pour développer une action bien éloignée de ce qu'attendaient les modernistes ; le jugement porté sur leur action par leurs collègues permet de mesurer l'écart entre ces deux groupes. Les critiques contre les bureaucrates émanent du groupe des innovateursprojet qui expriment l'idée que la démarche a été imposée et «non pas amenée en discussion et participation », que l'application du référentiel d'organisation a été trop stricte: «les gens l'ont appliquée en allant probablement trop loin [...]. Le résultat a été une hyperspécialisation qui allait probablement au-delà de la volonté recherchée qui était d'avoir des gens mieux formés, qui connaissaient mieux leur travail, la technique, etc. » Tous s'accordent pour dire que ce qui est en cause, ce n'est pas le référentiel mais son application, ce ne sont pas les règles mais leur usage stratégique par ce groupe «d'applicateurs zélés ». Les critiques aident à reconstituer ce groupe d'innovateurs-projet qui trouve ses origines dans « la détection faite par une hiérarchie qui ne voulait pas de vagues et qui cherchait des gens à son image ». Ce qui est en cause, ce n'est pas la démarche des modernistes, ni le référentiel sur lequel ils s'appuient, mais bien l'usage des règles : "la méthode était nécessaire, pas si mauvaise que ça, mais c'est l'utilisation qui en a été faite. »

Finalement, l'acteur collectif se construit face à d'autres acteurs qu'il tente de convaincre, d'enrôler. De manière symétrique les acteurs qui s'abritent derrière le système existant attendent, s'opposent ou freinent l'action collective au service du projet. Ce qui est clair c'est qu'ils n'interviennent pas dans la formation de nouvelles règles, mais tentent d'échapper à leurs conséquences, freinent leur formation ou leur application.

\section{Cognitif et pouvoir}

L'évolution des «manières de travailler» dans le secteur du Matériel s'est accompagnée d'une évolution dans les « manières de voir » l'entretien, qui s'est traduite par le passage d'un schéma d'organisation « en place » à un schéma d'organisation « émergent» que l'on propose d'interpréter comme un changement de référentiel : ce n'est pas seulement la dimension des pratiques, c'est-à-dire des espaces professionnels, de la nature des procédures de travail, de la ligne hiérarchique ou de la formation qui se modifie au cours de quarante années, mais aussi la manière de concevoirl'entretien, c'est-à-dire de définir le contexte des activités professionnelles, la nature des acteurs qui ont été mobilisés pour cela, les règles de décision de ce qui est une "procédure pertinente », les savoirs qui sont construits et combinés. 


\section{Une nouvelle "manière de concevoir " l'entretien portée par des acteurs sans pouvoir}

Dans la phase expérimentale, l'arrivée des machines nouvelles constitue une opportunité pour la création de savoirs pertinents permettant de résoudre le problème de leur entretien. Pour constituer ces savoirs, les détenteurs du projet de modernisation s'appuient sur les compagnons. En même temps qu'ils élaborent, pour définir les règles d'entretien, de nouvelles connaissances par observation du comportement des machines, les compagnons invalident les savoirs et les connaissances utilisées au temps de la vapeur par les «agents des méthodes » et qui servent de base pour le calcul des temps opératoires ; plus encore, ils remettent en question le monopole de la production normative, entièrement aux mains des experts, et montrent l'inefficacité de la centralisation des connaissances supposées détenues par les agents des méthodes. Les inventeurs s'appuient sur le niveau de compétence des compagnons pour qu'ils puissent désormais définir les normes pratiques : leur compétence acquiert une dimension normative ; en prenant l'initiative de produire des règles pratiques, les inventeurs affirment leur autonomie contre le pouvoir en place. Dans cette phase, initiative et pouvoir ne coïncident pas, mais l'initiative des inventeurs modifie les rapports de pouvoir et contient tous les ingrédients de ce qui va saper les fondements du pouvoir en place. Elle modifie les rapports de pouvoir, puisque désormais les compagnons ne sont plus obligés de cacher leur savoir-faire, de peur que les agents des méthodes n'augmentent les cadences ou par crainte de perdre les primes ; bien au contraire, ils affirment leurs compétences, les mettent au grand jour. De plus, cette initiative, de nature cognitive, conteste la légitimité des connaissances et la centralité du pouvoir normatif des experts ; les inventeurs délocalisent l'initiative vers le bas, décentralisent le pouvoir et font du compagnon l'acteur-clé de cette production de savoirs et de normes.

\section{L'élaboration d'une "doctrine " associée à une conquête du pouvoir}

Dans la phase de généralisation, les modernisateurs prennent l'initiative et élaborent «la doctrine » qui ne serait pas propagée sans la conquête du pouvoir central : l'initiative cognitive est inséparable de cette conquête du pouvoir. La «doctrine »", ce n'est pas seulement le

9- Nous utilisons ce terme, car il fait partie du langage de nos interlocuteurs, comme en témoigne l'entretien réalisé avec l'un des dirigeants de l'époque et les écrits d'un autre dirigeant (Quiniou, 1957). 
rappel des règles pratiques qu'il faut mettre en place concernant la documentation, la spécialisation ou la définition de la ligne hiérarchique. La doctrine, c'est la manière d'organiser les établissements à distance, comprenant tous les documents d'organisation : c'est une proposition d'un ensemble de règles d'action incomplètes, qu'il faut reprendre à son compte en les complétant, l'exemple le plus marquant est ce document d'organisation DO 205 stipulant que le bon modèle d'organisation n'existe pas, qu'il faut inventer à chaque fois une solution adaptée au contexte, qu'il faut laisser des marges de manœuvre pour faciliter l'implication des acteurs locaux. Des hommes sont détectés, formés pour constituer un corps de spécialistes au service de la doctrine. Enfin, les modernistes prennent le pouvoir et, en ce cas, le pouvoir coïncide avec la prise d'initiative, ce qui n'était pas le cas dans la phase précédente.

\section{Une conception de l'entretien par projet et un pouvoir distribué}

Dans la phase de différenciation, les innovateurs-projet vont reprendre l'initiative qu'ils estiment bloquée et vont défier le pouvoir des bureaucrates qui sont d'anciens modernistes ayant appliqué à la lettre la doctrine et l'ayant détournée de la voie tracée par ses concepteurs. Ils font, eux aussi, une lecture cognitive de la situation, ce qui leur permet d'établir la contre-performance des bureaucrates et de s'engager dans la formulation de projets spécifiques (qualité, raccourcissement de la ligne hiérarchique, etc.) : direction centrale et acteurs locaux d'établissements s'associent pour reprendre l'initiative et le pouvoir, en écartant les bureaucrates.

En conclusion, le pouvoir n'est pas une force d'imposition ; ce n'est pas celui d'une hiérarchie qui tenterait de contraindre ses subordonnés, puisque les acteurs se redistribuent selon une autre logique que celle de leur position et que le pouvoir se fonde sur une dimension cognitive, celle de la doctrine qui contient une force normative, liée à la réussite de la phase expérimentale. L'engagement et l'échange social constituent le moteur de ce travail d'organisation.

\section{Conclusion}

Que nous apprend la dynamique d'organisation de la maintenance du matériel roulant que nous venons de retracer de 1954 à 1994 ?

Premièrement, elle nous apprend qu'organiser la maintenance est une véritable activité qui nécessite de mobiliser des hommes, des compétences et des lieux de décisions pour être réalisée dans le sens préconisé. Cette activité d'organiser (Friedberg, 1995) ou organisatrice 
consiste à définir un cadre de travail, à formuler un ensemble de règles et à mettre en place une structure d'autorité et de communication, mais pas seulement. Ainsi, l'activité d'organisation formalise des espaces d'action en même temps qu'elle en ouvre, ce qui permet aux acteurs de s'en saisir et de se mêler des questions d'organisation. De plus, son développement relève davantage de conséquences inattendues compte tenu du jeu des acteurs : c'est un ensemble de pratiques instables, situées dans des contextes et résultant de rapports de force.

Deuxièmement, l'évolution de l'organisation du matériel roulant est entendue comme une construction sociale permanente qui concerne de multiples processus. Il y a d'abord un processus temporel d'initiatives que nous avons identifié sous la forme de trois phases : l'expérimentation, la généralisation, la différenciation. Il y a ensuite un processus cognitif qui concerne la construction d'une manière de voir l'entretien commune. Il y a un processus collectif qui concerne la construction d'un acteur collectif capable d'élaborer cette manière de voir et de la transformer en une manière de faire. Il y a un processus politique qui concerne le pouvoir et le système d'autorité qui vont réguler l'action organisationnelle. Enfin, il y a un processus d'apprentissage, résultat de l'articulation entre les savoirs mobilisés et les actions de maintenance qui se traduisent dans des règles et sont mémorisés par les collectifs.

Troisièmement, l'activité qui consiste à organiser la maintenance se présente comme une tentative permanente de synchronisation de ces cinq processus, au niveau d'initiatives inscrites dans un contexte temporel, au niveau cognitif, au niveau collectif, au niveau politique et au niveau de l'apprentissage. Chaque phase est caractérisée par une forme d'activité d'organiser spécifique qui combine ces processus. Ainsi, il y a différentes manières de produire des règles de maintenance au cours du temps. Pendant l'expérimentation, des savoirs nouveaux se construisent : ils vont arbitrer le choix des règles d'entretien (par exemple, la définition des cycles de visite) et mobiliser les acteurs (la formation d'un réseau d'hommes convaincus). Pendant la généralisation, la production de règles devient une véritable activité avec la constitution d'un acteur collectif (rapporteur, instructeur, binôme, encadrement) qui accepte de les produire. Pendant la différenciation, l'initiative de produire des règles change de camp et est reprise en main par les acteurs locaux qui s’en sentaient dépossédés.

L'analyse sociologique de l'histoire de l'organisation de la maintenance montre que l'organisation est une construction sociale dans laquelle l'ensemble des acteurs de la maintenance joue un rôle capital. Cette construction repose sur la combinaison de cinq processus 
fondamentaux qui régissent les dimensions constitutives de ce que nous avons appelé un « travail d'organisation ». De façon générale, on pourrait définir le TO comme la manière dont les acteurs, dans un contexte particulier, se mobilisent pour inventer des solutions singulières face à un problème particulier et pour mettre en place des dispositifs pour régler leurs échanges : le TO est une activité, l'activité de mobilisation et d'engagement pour résoudre un problème et pour gérer les interactions et les interdépendances entre les acteurs.

La perspective que nous avons présentée nous écarte des approches en terme de modèles d'organisation et nous conduit à considérer que la «bonne » organisation n'est pas celle qui est préconisée ou affichée, mais celle qu'inventent quotidiennement les acteurs, tant pour produire un service de qualité que pour faciliter leurs échanges : nous considérons le TO comme une activité de création de règles par laquelle les uns obligent les autres, mais tous s'obligent les uns les autres. Nous avons montré, d'une part, en quoi le TO est un travail de régulation au sens de la théorie de la régulation sociale élaborée par Reynaud (Reynaud, 1997 et 1999) et, d'autre part, quelle est la nature des régulations qu'inventent les acteurs pour travailler ensemble.

\section{Bibliographie}

- Friedberg, 1995 : Le Pouvoir et la règle, Paris, Seuil (1 ${ }^{\text {re }}$ édition 1993).

- J.-D., Reynaud, 1997 : Les Règles du jeu. L'action collective et la régulation sociale, Paris, A. Colin, $3^{\mathrm{e}}$ édition (1 $1^{\text {re }}$ édition, 1987).

- J.-D., Reynaud, 1999 : Le Conflit, la négociation et la règle, Toulouse, Octarès, (Recueil de textes, $1^{\text {re }}$ édition : 1995). 
